\title{
Computer-aided diagnosis of sonographic liver cirrhosis: A spleen-reference approach
}

\author{
Pei-Ming Yang \\ Department of Internal Medicine, College of Medicine, National Taiwan University, Taipei, Taiwan \\ and Division of Internal Medicine, National Taiwan University Hospital, Taipei, Taiwan
}

Chung-Ming Chen, ${ }^{\text {a) }}$ Tung-Wu Lu, and Chih-Pin Yen

Institute of Biomedical Engineering, National Taiwan University, Taipei, Taiwan

(Received 11 July 2007; revised 23 January 2008; accepted for publication 29 January 2008; published 6 March 2008)

This article aimed to develop a computer-aided diagnosis (CAD) algorithm for differential diagnosis of cirrhotic and noncirrhotic livers, allowing free adjustment of system settings to obtain the best scanned images. A spleen-referenced approached was proposed. 505 pairs of images acquired from 322 subjects using three ultrasound imaging systems were evaluated. Each pair consisted of liver and spleen sonograms of the same subject. Medical doctors were free to adjust the system parameters to attain the best scanned images. Of these 322 subjects, 155 subjects were cirrhotic, and 167 subjects noncirrhotic. Four assessments were carried out to evaluate the effectiveness and robustness of the proposed algorithm on the variations of regions-of-interest, ultrasound imaging systems, and scanned images. Four feature pairs were proposed. A logistic regression function was used as the classifier. All performances were evaluated by the leave-one-out cross-validation method. The proposed algorithm was compared with two previous CAD algorithms and all performance comparisons were based on paired-sample $t$ tests. The mean $A_{z}$ values of the receiver operating characteristic curves and classification accuracies ranged as high as from 0.949 to 0.996 and from $91.58 \%$ to $97.30 \%$, respectively, for the four assessments. At the 5\% family-wise significance level, the proposed algorithm was shown to be superior to the two previous CAD algorithms and their modified versions in terms of the $A_{z}$ values. (C) 2008 American Association of Physicists in Medicine. [DOI: 10.1118/1.2870217]

Key words: liver cirrhosis, ultrasound imaging, computer-aided diagnosis, watershed transform, logistic regression function, spleen

\section{INTRODUCTION}

Liver cirrhosis is a leading cause of death in many countries, especially in Asia. ${ }^{1,2}$ Because of its reasonably high accuracy and noninvasive nature, sonography has been widely used for the diagnosis of liver cirrhosis. To differentiate cirrhotic livers from noncirrhotic ones, various sonographic signs had been proposed, e.g., coarse echo textures, 3,4 nodular liver surfaces, ${ }^{5,6}$ irregular narrowing of the hepatic veins, ${ }^{3,7}$ etc. However, most sonographic signs are interpreter dependent. Since the interpretation heavily involves a subjective visual process and individualized knowledge and experience, different diagnoses might be made for the same sonography by different sonologists or by the same sonologist at different times.

To overcome the potential problems with liver sonography caused by its interpreter-dependent nature, many computerized approaches have been developed to assist the diagnosis of liver cirrhosis. The general idea of a computer-aided diagnosis (CAD) approach is to convert the sonographic signs into mathematical models and characterize liver cirrhosis with classification schemes based on these data. The advantage of using a CAD approach lies in its high reproducibility, resulting from the consistent quantitative criteria.

Most previous CAD algorithms for liver cirrhosis were based on hepatic echo textures. Some typical features char- acterizing hepatic textures were those from co-occurrence matrices, ${ }^{8-10}$ Fourier power spectrum, ${ }^{10}$ fractal descriptors, ${ }^{11}$ wavelet transformation, ${ }^{12}$ and so on. Although satisfactory performances have been repeatedly reported in previous studies, these CAD algorithms suffer two deficiencies that prevent them from being practically used. One is setting dependence and the other is person dependence.

It can be easily shown that the values of many conventional texture-based mathematical features vary with the parameter settings of an ultrasound imaging system. For instance, the entropy, contrast, and correlation derived from a co-occurrence matrix ${ }^{8}$ might fluctuate with such parameters as the time-gain compensation (TGC), total gain, and scanning depth. To overcome setting-dependence, many previous CAD algorithms ${ }^{9,11}$ required that all hepatic ultrasound images be acquired with the same parameter settings, which was definitely an undesirable constraint in practical use. Alternatively, several approaches have been proposed to achieve setting-independence based on ultrasonic radio frequency (RF) signals. ${ }^{13-16}$ By carefully correcting the effects of TGC, beam diffraction, beam focusing, and attenuation on the RF signals, the mathematical features extracted from the RF signals are, at least theoretically, setting independent. Promising as they were initially, these approaches have remained impractical because of their inadequate performance 
on differentiating cirrhotic and noncirrhotic livers. Moreover, the limited access to RF signals for most users is the major obstacle to the further improvement of this class of approaches.

Person-dependence refers to the fact that different persons with normal livers might have different degrees of hepatic texture coarseness. Even with standardized parameter settings or RF signals, it is not unusual to see a normal liver with coarse echo textures or signals. Therefore, hepatic echo textures or RF signals alone might not be sufficient for diagnosis of liver cirrhosis due to the person-dependent property of the tissue.

This study aimed to develop a texture-based CAD algorithm for diagnosis of liver cirrhosis which not only would allow medical doctors to freely adjust the system settings to acquire the best views but also would account for the potential variations in the echo textures of normal livers. Moreover, the proposed CAD algorithm was designed to work on sonograms acquired from various ultrasound imaging systems of different models.

\section{MATERIALS AND METHODS}

To minimize the potential setting and person dependence, the essential idea of the proposed CAD algorithm was to define each mathematical feature as a feature pair, i.e., a two-element feature vector. These two elements characterized the same sort of sonographic signs, one for hepatic echo texture and the other for splenic echo texture. The splenic feature element in a feature pair served as a reference to account for the potential variations in hepatic echo textures caused by different system settings and personal texture properties. This idea originated from the clinical practice proposed by Yang $e t$ al. ${ }^{3}$ based on two assumptions. One was that the echo textures of the normal liver and spleen of an individual had similar texture properties. The other was that both liver and spleen sonograms were acquired with the same parameter settings.

Based on the spleen-referenced approach, four mathematical feature pairs were proposed to characterize the coarseness and homogeneity of hepatic and splenic echo textures. The classifier was a logistic regression function ${ }^{16}$ using all four feature pairs together as the predictor variables. Liver cirrhosis was confirmed either by biopsy or based on clinical evidences. If a biopsy was performed, in this study, a case was considered as liver cirrhosis if its Ishak's modified histological activity index score ${ }^{17}$ was 5 or 6 and as noncirrhotic if the score was 0 or 1 . On the other hand, if no biopsy was carried out, a liver cirrhosis case was diagnosed by experienced physicians based on clinical evidences, including liver sonograms and such evidences as ascites, esophageal varices detected in panendoscopic examination, and so on. A noncirrhotic case was one without the support of sonographic signs or clinical evidence of liver fibrosis and cirrhosis. A noncirrhotic case might or might not be a case of fatty liver in this study.

\section{II.A. Study subjects and image acquisition}

Three ultrasound imaging systems were used to capture the liver and spleen sonograms to evaluate the robustness of the proposed CAD algorithm on different machines. These three systems were the Toshiba SSA-370A, Toshiba SSA380A (Toshiba Medical System Corporation, Tochigi-ken, Japan) and Aloka SSD-4000 (Aloka, Inc., Tokyo, Japan). Table I summarizes the numbers of cirrhotic and noncirrhotic subjects imaged by each system, the numbers of images per subject for each system, and the bases on which the diagnosis of either cirrhosis or non-irrhosis was made. Among these subjects, 30 cirrhotic and 30 noncirrhotic subjects imaged by Toshiba SSA-380A were randomly selected as the learning set, which was employed to determine the parameter values involved in the extraction of the proposed features. The rest of the subjects formed the test set for performance evaluation of all algorithms. In addition, Table I also shows how the images were acquired as well as the transducer and its operating frequency for each system. The frame grabber card used by the Toshiba SSA-380A was the Meteor-II card (Matrox Electronic System, Ltd., Quebec, Canada). All images were collected from a teaching hospital in Taiwan by at least ten medical doctors from August 10, 1998 to February 14, 2006. The ages of the subjects ranged from 13 to 81 years, the first, second, and third quartiles of which were 42, 53, and 62 , respectively. More detailed demographic data were listed in Table I. The Institutional Review Boards agreed that the patients' images could be used for study without written consent if the patients' privacy could be well protected. This regulation has been carefully followed in this study.

The medical doctors were free to adjust the parameters of the ultrasound imaging system to obtain the best scanned images according to their own experience, but the liver and spleen images of the same pair were obtained with the same parameter settings. The hepatic and splenic feature elements in a mathematical feature pair were extracted from the regions-of-interest (ROIs) of the same size for each pair of liver and spleen sonograms. The sizes of the ROIs might be different for different pairs of liver and spleen sonograms. The mean and standard deviation of all ROI sizes were 2713 and 1272 pixels, respectively. The depths of the ROIs from the top of the images were designated to be the same for each pair of liver and spleen sonograms. To take into account the potential variation of mathematical features due to the selection of the ROIs, four sets of ROIs were selected for each pair of liver and spleen sonograms in the test set, which were performed by four graduate students, each for one set. For the learning set, only one ROI was selected for each sonogram. All students were trained to avoid vascular structures and artifacts in selecting ROIs.

\section{II.B. Proposed mathematical features}

Two types of mathematical feature pairs were devised to characterize the coarseness and homogeneity of the hepatic and splenic echo textures of the same person. The basic idea was to highlight three evident phenomena frequently observed in the liver and spleen sonograms of the subjects with 
TABLE I. Summaries of the study subjects and image acquisition for Toshiba SSA-370A, Toshiba SSA-380A, and Aloka SSD-4000, respectively, where for each subgroup, $N_{M}$ and $N_{F}$ stand for the number of male and female subjects, respectively, $m 1, m 2$, and $m 3$ stand for the first, second, and third quartiles of the ages of the male subjects, respectively, $f 1, f 2$, and $f 3$ stand for the first, second, and third quartiles of the ages of the female subjects, respectively.

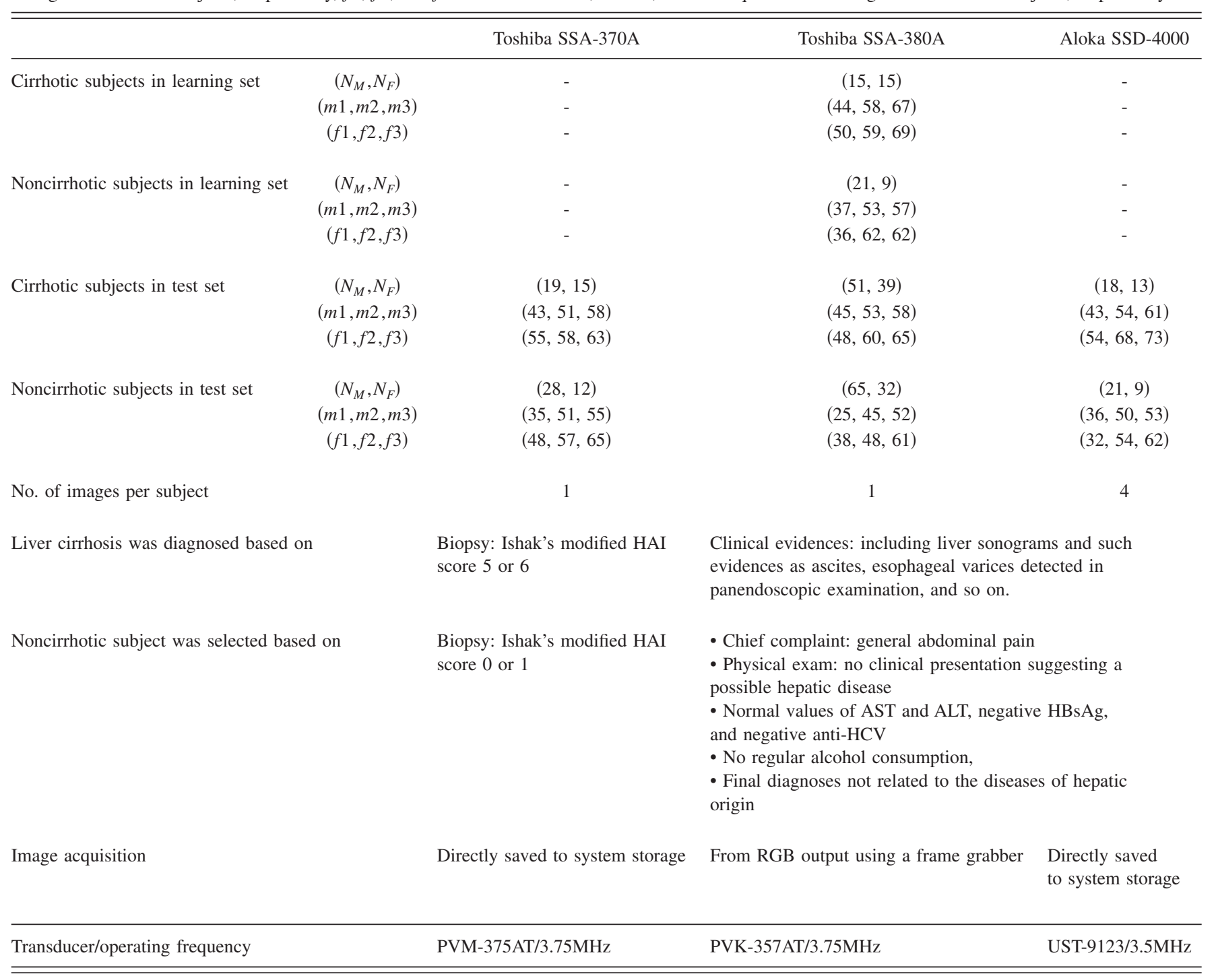

liver cirrhosis. That is, the echo texture of a cirrhotic liver usually appeared to be more heterogeneous, have more highcontrast spots, and have more large texture structures than the splenic echo texture of the same person. These two types of feature pairs were region-based features and texton-based features designed to quantify the first and the last two phenomena, respectively. Perceptually, a sonogram might be regarded as a composition of slowly varying areas superimposed with grain-like texture elements, called textons, formed by the speckles and tissue-related textures.

The procedures to obtain these feature pairs are sketched in Fig. 1. Each block is labeled at the upper-left corner for ease of reference. Several parameters were involved in the derivation of these feature pairs. The parameter values were determined empirically by evaluating a range of values for each feature pair. Evaluation was performed separately for each feature pair using the learning set. Based on the leaveone-out cross-validation method, the parameter values yielding the largest $A_{z}$ value, i.e., the area under the receiver op- erating characteristic curve (ROC curve), for this feature pair were used for all images and ROIs. Table II summarizes the proposed region- and texton-based feature pairs, their definitions, and the parameter values determined by the learning set.

\section{II.B.1. Region-based features}

The region-based features were designed to estimate the granularity and homogeneity of the liver and spleen sonograms in terms of the average sizes of homogeneous regions. The homogeneity of a region was defined based on an empirical model that the ratio between the variance and mean in a sufficiently large uniform area, e.g., $11 \times 11$ pixels, is roughly a constant on a displayed ultrasound image. ${ }^{18-22} \mathrm{~A}$ homogeneous region thus might be defined as a connected region in which all pixels have approximately the same variance-to-mean (VM) ratios. 


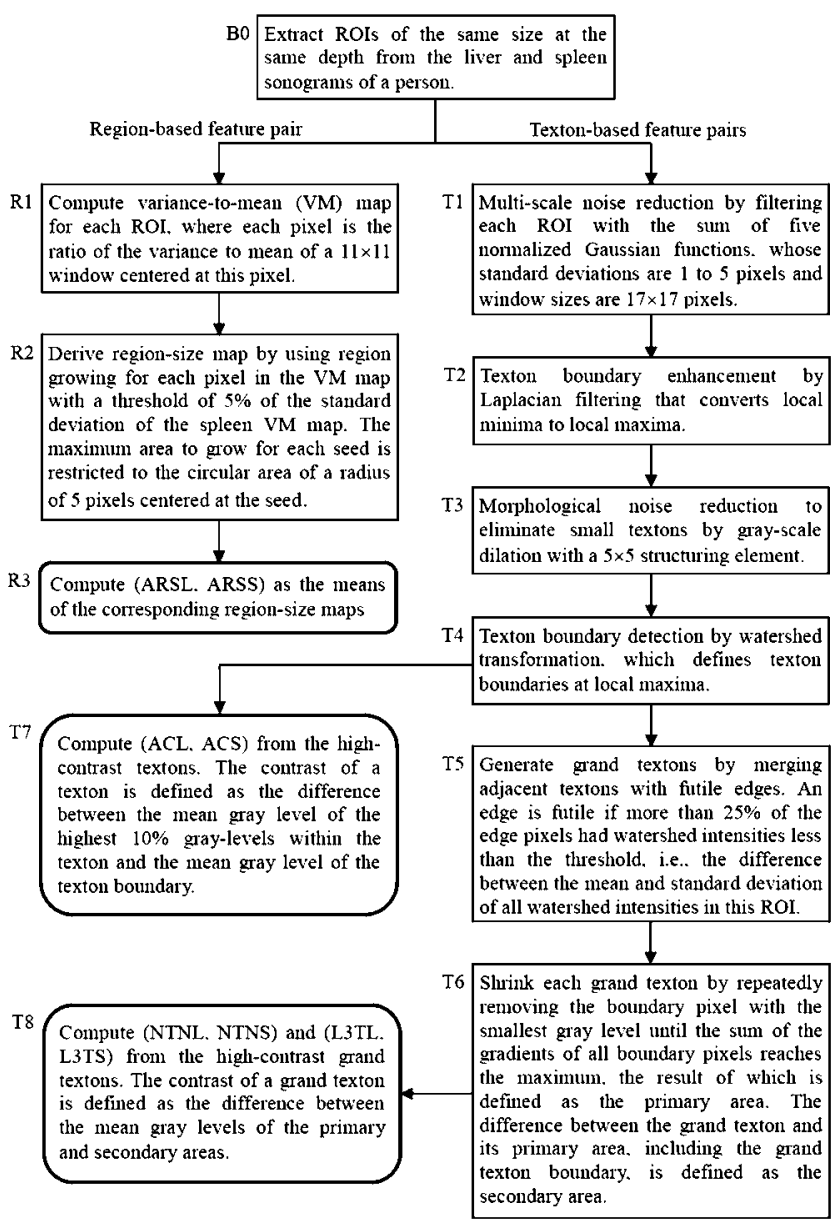

FIG. 1. The flowchart for the procedures of feature extraction, the left and right branches of which were for extracting the region-based and textonbased feature pairs, respectively.

One region-based mathematical feature pair, denoted by (ARSL, ARSS), was derived based on the empirical model as defined in Table II. The steps to derive ARSL and ARSS are described in blocks R1-R3 of Fig. 1. Following Karaman et al., ${ }^{19}$ the window size was set to $11 \times 11$ pixels as given in block R1.

\section{II.B.2. Texton-based features}

The gray-level profile of a ROI may be viewed as a threedimensional landscape composed of plains, elevated areas of land, e.g., hills, plateaus, etc., and low areas of lands, e.g., valleys, gorges, ravines, etc. In this study, a texton is defined as the area covered by an elevated area of land enclosed by the nearest local minima. When the low area of land in between two adjacent textons is relatively negligible because of the low contrast between the texton boundaries and the local maxima of textons, these adjacent textons might be further grouped into a grand texton corresponding to a large visually perceptible texture structure. The texton and grand texton play different roles in characterizing echo textures. The texton was devised to identify the high-contrast spots, since the gray level profile of a high-contrast spot in a cirrhotic liver sonogram usually appears to be a steep hill or mountain and has a clear gray-level difference in its vicinity. The grand texton might be used to describe a large visually perceptible texture structure, the gray-level profile of which is usually composed of multiple stretched hills or mountains.

Identification of the textons and grand textons was the key step toward texton-based feature extraction. As shown in Fig. 1, four essential tasks (blocks T1-T4) were involved in identifying the texton boundaries in a ROI, followed by a merging process to obtain the grand textons (blocks T5 and T6). For example, Figs. 2(a) and 2(b) show the ROIs of the liver and spleen sonograms of the same person, respectively. By using multiscale Gaussian filtering, multiscale noise reduction (block T1) aimed to reduce noises while preserving the desired structures of various resolutions. Following that, texton boundary enhancement (block T2) was to transform the nonflat elevated and the low areas of lands of the smoothed gray-level profiles into the low and elevated areas of lands, respectively. To eliminate the small low areas of land, morphological noise reduction (block T3) was applied after texton boundary enhancement. The texton boundaries were then defined by the watersheds using the immersion method ${ }^{22}$ (block T4). For example, the texton boundaries defined at the watersheds for Figs. 2(a) and 2(b) were delineated with gray contours in Figs. 2(c) and 2(d), respectively.

TABLE II. The proposed region-based and texton-based feature pairs, including the names and definitions of the feature pairs and the parameter values determined by the learning set.

\begin{tabular}{|c|c|c|c|}
\hline Feature type & Feature name & Feature definitions & Parameter values determined by the learning set \\
\hline Region-based & (ARSL, ARSS) & $\begin{array}{l}\text { ARSL and ARSS were the average region sizes of the } \\
\text { liver and spleen sonograms of the same person, } \\
\text { respectively. }\end{array}$ & The threshold and maximum growing radius $(\mathrm{R} 2)^{\mathrm{a}}$ \\
\hline \multirow[t]{3}{*}{ Texton-based } & (ACL, ACS) & $\begin{array}{l}\text { ACL and ACS were the average of the top } 50 \% \text { of the } \\
\text { contrasts for liver and spleen ROIs, respectively. }\end{array}$ & $\begin{array}{l}\text { The standard deviation and window size of Gaussian } \\
\text { functions (T1), and the structuring element size (T3). }\end{array}$ \\
\hline & (NTNL, NTNS) & $\begin{array}{l}\text { NTNL and NTNS were the number of selected grand } \\
\text { textons in the ROIs of the liver and spleen sonograms, } \\
\text { respectively, divided by the pixel numbers in the ROIs. }\end{array}$ & \\
\hline & (L3TL, L3TS) & $\begin{array}{l}\text { L3TL and L3TS were the mean sizes of the largest } \\
\text { three selected grand textons in the ROIs of the liver and } \\
\text { spleen sonograms, respectively. }\end{array}$ & \\
\hline
\end{tabular}

${ }^{\mathrm{a}}$ Block label in Fig. 1. 


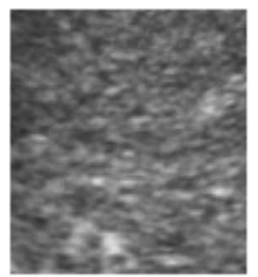

(a)

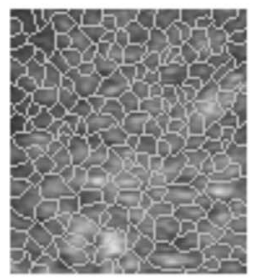

(c)

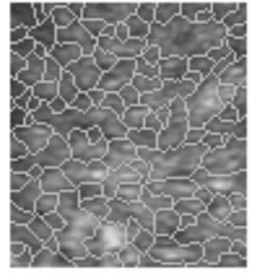

(e)

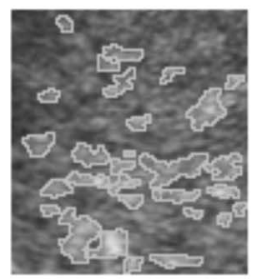

(g)

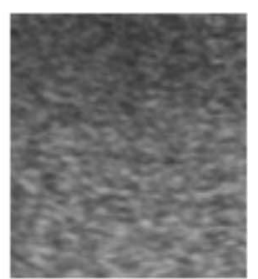

(b)

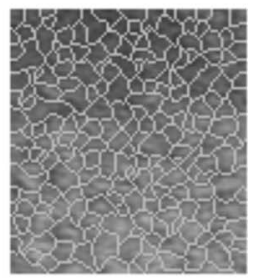

(d)

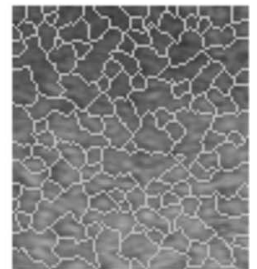

(f)

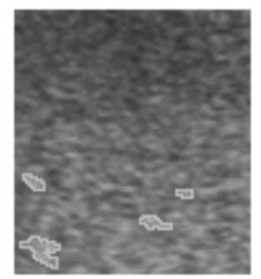

(h)
FIG. 2. (a) and (b) the ROIs of the liver and spleen sonograms of the same person, respectively, both of which were the same size and were selected from the same depth of the liver and spleen sonograms (block B0 of Fig. 1); (c) and (d) the texton boundaries in gray contours defined at the watersheds for Figs. 2(a) and 2(b), respectively (block T4 of Fig. 1); (e) and (f) the grand textons derived by merging textons in Figs. 2(c) and 2(d), respectively (block T5 of Fig. 1); and (g) and (h) the selected grand textons of the liver and spleen ROIs derived from Figs. 2(e) and 2(f), respectively (block T8 of Fig. 1).

The grand textons of a ROI were generated by merging the adjacent textons with futile edges (block T5). An edge is futile if more than $25 \%$ of the edge pixels had watershed intensities less than the threshold, i.e., the difference between the mean and standard deviation of all watershed intensities in this ROI. Figures 2(e) and 2(f) show the grand textons derived by merging textons in Figs. 2(c) and 2(d), respectively. Clearly, some large visually perceptible texture structures initially divided into several textons have been merged into integral ones, e.g., those large texture structures in the lower part of Fig. 2(e).

Three texton-based mathematical feature pairs (blocks T7 and T8) were extracted from the ROIs resulted from blocks T4, T5, and T6. The contrast of a texton was defined as the difference between the mean gray level of the highest $10 \%$ gray levels within the texton and the mean gray level of the texton boundary. As defined in Table II, the first mathematical feature pair (T7), denoted as (ACL, ACS), was designed to estimate the richness of high-contrast textons.

The second and third mathematical feature pairs (T8) were intended to describe the number and size, respectively, of the large visually perceptible texture structures represented by the grand textons in a ROI. Although each grand texton could reasonably demarcate a visually perceptible texture structure, the area enclosed by a grand texton usually looked larger than the perceptible size of the corresponding texture structure. To represent a visually perceptible texture structure more closely, each grand texton was further shrunk by repeatedly removing the boundary pixel with the smallest gray level until the sum of the gradients of all boundary pixels reached the maximum (block T6). Each grand texton was then divided into two areas. The area inside the shrunk version of the grand texton, excluding the boundary of the shrunk version, was defined as the primary area. The area outside the shrunk version but inside the grand texton, including the boundary of the original grand texton, was defined as the secondary area. The size of a grand texton was then defined as the number of pixels in the primary area of the grand texton. The contrast of a grand texton was defined as the difference between the mean gray levels of the primary and secondary areas.

The second mathematical feature pair, denoted as (NTNL, NTNS), and the third mathematical feature pair, denoted as (L3TL, L3TS), were defined on the basis of those selected grand textons with contrasts greater than the mean contrast of all grand textons in the liver ROI. The definitions of both feature pairs were given in Table II. As examples, Figs. 2(g) and 2(h) showed the selected grand textons of the liver and spleen ROIs derived from Figs. 2(e) and 2(f), respectively.

\section{II.C. Comparative studies}

To demonstrate the advantage of the spleen-referenced approach, the proposed CAD algorithm was compared with two previous CAD algorithms, namely the Ogawa algorithm ${ }^{10}$ and the Yeh algorithm. ${ }^{23}$ Both algorithms used only liver sonograms for feature extraction. In the Ogawa algorithm, five ROIs were selected from each liver sonogram, including one center ROI and four neighboring ROIs. The size of each ROI was $32 \times 32$ pixels. Each of the four neighboring ROIs overlapped with half of the center ROI. Seven features were extracted in the Ogawa algorithm. The first six features were computed from the center ROI, including variance, coefficient of variation, annular Fourier power spectrum, longitudinal Fourier power spectrum, angular second moment, and contrast of the co-occurrence matrix with $(d, \theta)=\left(1,0^{\circ}\right)$. The seventh feature was the variation of the means computed from all five ROIs. The classifier used in the Ogawa algorithm was a three-layer artificial neural network with seven neurons in the hidden layer.

For the Yeh algorithm, the six features resulting in the best performance in Yeh et al. ${ }^{23}$ were used in this study, including five gray level co-occurrence (GLC) matrix features and the variance of the second-level high-pass filtered 
image using the nonseparable quincunx wavelet transformation. ${ }^{12}$ Each ROI was a square subimage composed of $64 \times 64$ pixels from the liver sonogram. The five GLC features were angular second moment, contrast, correlation, entropy and sum entropy. The classifier used in the Yeh algorithm was a support vector machine with a Gaussian radial-basis function.

To make the performance comparison as fair as possible, we tried to maximize the overlapping areas of the ROIs selected for the proposed algorithm and those for the Ogawa algorithm and the Yeh algorithm. Our strategy was to locate the ROIs of the Ogawa algorithm and the Yeh algorithm as close to the center of the ROIs of the proposed algorithm as possible, subject to the constraint of avoiding areas of vascular structures and artifacts. Furthermore, since all three algorithms used different classifiers, to compare the effectiveness of the mathematical features, both the Ogawa algorithm and the Yeh algorithm were modified by using logistic regression functions as the classifiers. Both the original and modified versions of the Ogawa algorithm and the Yeh algorithm were evaluated in this study.

\section{II.D. Performance analyses}

To show the potential advantage of using the computer to assist diagnosis of liver cirrhosis based on ultrasound images, two attending physicians were asked to independently classify all images used in this study into three categories: cirrhotic, noncirrhotic, and equivocal. These two attending physicians had 1 (observer A) and 5 years (observer B) of experience in liver sonography. Four performance figures were measured, namely, accuracy, sensitivity, specificity and interobserver variability, where true positive meant a cirrhotic liver was considered cirrhotic. The interobserver variability was the percentage of images for which the attending physicians made different classifications.

The performances of all algorithms were estimated based on the leave-one-out cross-validation method. For the proposed algorithm, two sets of performance indices were reported for each analysis. The first set consisted of the best classification accuracy as well as the associated sensitivity, specificity, positive predictive value (PPV), and negative predictive value (NPV). The second set included the $A_{z}$ value. For the other algorithms, for conciseness, only the best classification accuracy, sensitivity, specificity, and the $A_{z}$ value were reported. The performance of each algorithm was analyzed in terms of its effectiveness and robustness. The effectiveness of an algorithm referred to the mean $A_{z}$ value and the mean best classification accuracy attained in an assessment. The robustness of an algorithm referred to the variation caused by the factor evaluated in an assessment, which was defined as the coefficient of variation $(\mathrm{CV})$ of the four $A_{z}$ values achieved.

To account for the effects of the ROI, image, and system, four assessments were carried out for each CAD algorithm. Assessment I was designed to assess the effect of the ROIs on the performance of each algorithm given an ultrasound imaging system, which was evaluated by using four ROIs selected by four people for each image of a system. Assessment II was to assess the effect of different systems on the CAD performance. The emphasis would be on if the performance of each CAD algorithm would vary substantially with systems. In assessment III, the ROIs of the images acquired from all three systems, one image per subject, were pooled together to form four pooled sets of ROIs. The $i$ th pooled set of the ROIs comprised the $i$ th ROI of each image. This assessment evaluated the effectiveness and robustness of each CAD algorithm on a set of ROIs with a heterogeneous (system-dependent) nature of echo texture. By using the Aloka SSD-4000, assessment IV aimed to show the effect of scanned images on each CAD algorithm by acquiring four scanned images of different locations for each person and using only one ROI for each image.

Five paired-sample $t$ tests were carried out for performance comparisons between the proposed CAD algorithm and each of the other four algorithms. The first three tested if the proposed algorithm outperformed the algorithm being compared for each of the three systems. The performance figures used in these three tests were those derived in assessments I and II. Using the performance figures attained in assessment III, the fourth paired-sample $t$ test was to test if the proposed algorithm was superior to its counterpart for the pooled sets of ROIs. By using the performance figures of assessment IV, the fifth paired-sample $t$ test was to test if the proposed algorithm was better than the algorithm being compared when using four scanned images acquired from the Aloka SSD-4000 for each subject. All paired-sample $t$ tests were one-tailed tests based on the $A_{z}$ values of the ROC curves. Since five tests were involved in the comparison between two algorithms, the overall type I error rate would be greater than the significance level of each test. To control the overall type I error rate, i.e., the family-wise type I error rate, the family-wise significance level $\alpha$ was set to 0.05 , and the individual significance level of each paired-sample $t$ test $\alpha^{\prime}$ was set to $1-(1-0.05)^{1 / 5}=0.0102$.

\section{RESULTS}

The number of images classified by each attending physician was 505. Table III summarizes the classification results made by the two attending physicians (i.e., observers $\mathrm{A}$ and B) for the three systems In Table III, TP, TN, FP, and FN stand for true positive, true negative, false positive, and false negative, respectively, and "equivocal" refers to a case in which an image could not be classified as either cirrhotic or noncirrhotic by the observer. The observer accuracies, sensitivities, and specificities achieved by each observer for each individual system and for all three systems are also listed in Table III. The percentages of images that observers A and B classified as equivocal were $13.07 \%$ and $12.48 \%$, respectively. The interobserver variability was $24.36 \%$.

The performance figures achieved by each algorithm in the four assessments on three ultrasound imaging systems are summarized in Tables IV and V. For conciseness, each set of four performance figures is represented by the mean and standard deviations of these four figures. The robustness 
TABLE III. Classification performances achieved by the two attending physicians (observers A and B) for 505 pairs of liver and spleen sonograms

\begin{tabular}{|c|c|c|c|c|c|c|}
\hline \multirow{2}{*}{$\begin{array}{l}\text { Performance } \\
\text { figures }^{\mathrm{a}}\end{array}$} & \multicolumn{3}{|c|}{ Observer A } & \multicolumn{3}{|c|}{ Observer B } \\
\hline & Toshiba 370A & Toshiba 380A & Aloka 4000 & Toshiba 370A & Toshiba 380A & Aloka 4000 \\
\hline $\mathrm{TP} / \mathrm{TN}$ & & $174 / 224$ & & & $185 / 221$ & \\
\hline $\mathrm{FP} / \mathrm{FN}$ & & $3 / 38$ & & & $7 / 29$ & \\
\hline Equivocal & & 66 & & & 63 & \\
\hline Overall accu (\%) & & 78.81 & & & 80.40 & \\
\hline Overall sens $(\%)$ & & 70.16 & & & 74.60 & \\
\hline Overall spec (\%) & & 87.16 & & & 85.99 & \\
\hline $\mathrm{TP} / \mathrm{TN}$ & $29 / 29$ & $60 / 87$ & $85 / 108$ & $24 / 36$ & $75 / 77$ & $86 / 108$ \\
\hline $\mathrm{FP} / \mathrm{FN}$ & $0 / 3$ & $2 / 14$ & $1 / 21$ & $0 / 3$ & $6 / 3$ & $1 / 23$ \\
\hline Equivocal & 13 & 24 & 29 & 11 & 26 & 26 \\
\hline Accu $(\%)$ & 78.38 & 78.61 & 79.10 & 81.08 & 81.28 & 79.51 \\
\hline Sens $(\%)$ & 85.29 & 66.67 & 68.55 & 70.59 & 83.33 & 69.36 \\
\hline Spec $(\%)$ & 72.50 & 89.69 & 90.00 & 90.00 & 79.38 & 90.00 \\
\hline
\end{tabular}

${ }^{\mathrm{a}}$ Definitions of performance figures. TP, TN, FP, and FN: the numbers of true positive, true negative, false positive, and false negative predicted by each observer, respectively; equivocal: an image could not be classified as either cirrhotic or noncirrhotic by an observer; Accu: abbreviation of accuracy $=(\mathrm{TP}+\mathrm{TN}) /(\mathrm{TP}+\mathrm{TN}+\mathrm{FP}$ $+\mathrm{FN}+$ equivocal); Sens: abbreviation of sensitivity $=\mathrm{TP} /$ the number of cirrhotic images acquired from a system or all systems; and Spec: abbreviation of specificity $=\mathrm{TN} /$ the number of noncirrhotic images acquired from a system or all systems.

of each algorithm to the factor evaluated in each assessment is provided in Table VI in terms of CV values. The superiority of the proposed CAD algorithm to the other four approaches was validated by the results of paired-sample $t$ tests. The null hypothesis for each test was that the mean $A_{z}$ value of the proposed algorithm would be less than or equal to that of the algorithm being compared in an assessment. The $p$ values for most one-tailed paired-sample $t$ tests are less than the individual significance level, $\alpha^{\prime}=0.0102$. That is, the null hypothesis was rejected for most cases. The only two exceptions are the test results when comparing the proposed algorithm with the original and modified versions of the Yeh algorithm on the Aloka-4000 using one image per subject and four ROIs per image, the $p$ values of which are 0.026 and 0.017 , respectively.
The superior performances achieved by the proposed CAD algorithm were partly due to the effectiveness of the new texture features found in this study and partly due to the incorporation of splenic features as references. To demonstrate these two points, for conciseness, we used assessment III as an example because the images were from three different imaging systems, which gave images with quite heterogeneous image properties. If the same set of proposed hepatic features was used for each set of ROIs, i.e., without incorporating splenic features, the mean $A_{z}$ value and classification accuracy were $0.932 \pm 0.007$ and $(86.34 \pm 1.70) \%$. It is clear that using the proposed hepatic features alone also outperformed the original and modified versions of the Ogawa algorithm and the Yeh algorithm at the 5\% significance level, which supports the effectiveness of the proposed

TABLE IV. Performances of the proposed CAD algorithm achieved in the four assessments on three ultrasound imaging systems, where $a \pm b$, shown in each cell, stands for the mean $a$ and standard deviation $b$ of each performance figure. Assessment I: assessing the effect of ROIs on each algorithm. Assessment II: assessing the effect of different systems on the CAD performance. Assessment III: assessing the effectiveness and robustness of each CAD algorithm on a set of ROIs with a heterogeneous (system-dependent) nature of echo texture. Assessment IV: assessing the effect of scanned images on each CAD algorithm.

\begin{tabular}{|c|c|c|c|c|c|}
\hline \multirow[b]{2}{*}{ Performance figures ${ }^{\mathrm{a}}$} & \multicolumn{3}{|c|}{ Assessments I and II } & \multirow{2}{*}{$\frac{\text { Assessment III }}{\text { All systems }}$} & \multirow{2}{*}{$\frac{\text { Assessment IV }}{\text { Aloka-4000 }}$} \\
\hline & Toshiba-370A & Toshiba-380A & Aloka-4000 & & \\
\hline $\operatorname{ROC} A_{z}$ & $0.996 \pm 0.003$ & $0.949 \pm 0.012$ & $0.974 \pm 0.027$ & $0.965 \pm 0.007$ & $0.975 \pm 0.008$ \\
\hline Accuracy $(\%)$ & $97.30 \pm 1.10$ & $91.58 \pm 0.80$ & $95.08 \pm 3.54$ & $92.70 \pm 1.31$ & $95.08 \pm 1.34$ \\
\hline Sensitivity $(\%)$ & $96.32 \pm 3.70$ & $88.89 \pm 2.40$ & $95.97 \pm 3.09$ & $90.00 \pm 1.71$ & $93.55 \pm 2.63$ \\
\hline $\operatorname{Specificity}(\%)$ & $98.13 \pm 2.39$ & $94.07 \pm 3.08$ & $94.17 \pm 5.00$ & $95.21 \pm 3.17$ & $96.67 \pm 2.72$ \\
\hline PPV (\%) & $97.88 \pm 2.67$ & $93.42 \pm 2.99$ & $94.55 \pm 4.58$ & $94.69 \pm 3.41$ & $96.74 \pm 2.56$ \\
\hline NPV $(\%)$ & $97.02 \pm 2.91$ & $90.18 \pm 1.73$ & $95.77 \pm 3.18$ & $91.14 \pm 1.25$ & $93.62 \pm 2.42$ \\
\hline
\end{tabular}

${ }^{\mathrm{a}}$ Definitions of performance figures. TP, TN, FP, and FN: the numbers of true positive, true negative, false positive, and false negative predicted by the proposed algorithm, respectively; ROC $A_{z}$ : the area under the ROC curve; accuracy $=(\mathrm{TP}+\mathrm{TN}) /(\mathrm{TP}+\mathrm{TN}+\mathrm{FP}+\mathrm{FN})$; sensitivity $=\mathrm{TP} /(\mathrm{TP}+\mathrm{FN}) ;$ specificity $=\mathrm{TN} /(\mathrm{TN}+\mathrm{FP}) ; \mathrm{PPV}$ $=\mathrm{TP} /(\mathrm{TP}+\mathrm{FP})$; and $\mathrm{NPV}=\mathrm{TN} /(\mathrm{TN}+\mathrm{FN})$. 
TABLE V. The $A_{z}$ values and the best classification accuracies of the original and modified versions of the Ogawa algorithm and the Yeh algorithm achieved in the four assessments on the three ultrasound imaging systems, where $a \pm b$, shown in each cell, stands for the mean $a$ and standard deviation $b$ of the four performance figures.

\begin{tabular}{|c|c|c|c|c|c|c|}
\hline \multirow[b]{2}{*}{ Algorithms } & \multirow{2}{*}{$\begin{array}{l}\text { Performance } \\
\text { figures }^{\mathrm{a}}\end{array}$} & \multicolumn{3}{|c|}{ Assessments I and II } & \multirow{2}{*}{$\frac{\text { Assessment III }}{\text { All systems }}$} & \multirow{2}{*}{$\frac{\text { Assessment IV }}{\text { Aloka-4000 }}$} \\
\hline & & Toshiba-370A & Toshiba-380A & Aloka-4000 & & \\
\hline \multirow[t]{4}{*}{ Ogawa } & $\operatorname{ROC} A_{z}$ & $0.919 \pm 0.033$ & $0.891 \pm 0.014$ & $0.885 \pm 0.026$ & $0.844 \pm 0.032$ & $0.885 \pm 0.024$ \\
\hline & Accu $(\%)$ & $87.16 \pm 2.59$ & $83.56 \pm 1.01$ & $85.66 \pm 2.80$ & $79.50 \pm 3.12$ & $84.02 \pm 5.42$ \\
\hline & Sens $(\%)$ & $86.76 \pm 3.80$ & $81.94 \pm 2.78$ & $79.84 \pm 7.15$ & $73.06 \pm 7.04$ & $86.29 \pm 9.27$ \\
\hline & $\operatorname{Spec}(\%)$ & $87.50 \pm 6.12$ & $85.05 \pm 1.03$ & $91.67 \pm 5.77$ & $85.48 \pm 7.24$ & $81.67 \pm 15.52$ \\
\hline \multirow{4}{*}{$\begin{array}{l}\text { Modified } \\
\text { Ogawa }\end{array}$} & $\operatorname{ROC} A_{z}$ & $0.894 \pm 0.020$ & $0.870 \pm 0.006$ & $0.888 \pm 0.024$ & $0.867 \pm 0.010$ & $0.898 \pm 0.023$ \\
\hline & Accu $(\%)$ & $84.80 \pm 0.68$ & $83.42 \pm 0.44$ & $84.84 \pm 2.46$ & $81.68 \pm 1.08$ & $84.43 \pm 3.90$ \\
\hline & Sens $(\%)$ & $81.62 \pm 6.95$ & $80.28 \pm 5.00$ & $82.26 \pm 5.59$ & $73.87 \pm 4.23$ & $81.45 \pm 7.15$ \\
\hline & Spec $(\%)$ & $87.50 \pm 6.12$ & $86.34 \pm 4.64$ & $87.50 \pm 7.39$ & $88.92 \pm 2.70$ & $87.50 \pm 5.69$ \\
\hline \multirow[t]{4}{*}{ Yeh } & $\operatorname{ROC} A_{z}$ & $0.871 \pm 0.040$ & $0.875 \pm 0.014$ & $0.896 \pm 0.034$ & $0.885 \pm 0.015$ & $0.875 \pm 0.037$ \\
\hline & Accu $(\%)$ & $83.78 \pm 3.49$ & $80.75 \pm 2.05$ & $86.07 \pm 3.90$ & $81.91 \pm 1.25$ & $83.20 \pm 4.71$ \\
\hline & Sens $(\%)$ & $68.38 \pm 6.52$ & $88.33 \pm 4.30$ & $80.65 \pm 6.97$ & $80.00 \pm 2.93$ & $84.68 \pm 8.48$ \\
\hline & Spec $(\%)$ & $96.88 \pm 3.15$ & $73.71 \pm 5.98$ & $91.67 \pm 3.33$ & $83.68 \pm 1.98$ & $81.67 \pm 10.36$ \\
\hline \multirow{4}{*}{$\begin{array}{l}\text { Modified } \\
\text { Yeh }\end{array}$} & $\operatorname{ROC} A_{z}$ & $0.888 \pm 0.016$ & $0.889 \pm 0.019$ & $0.877 \pm 0.033$ & $0.885 \pm 0.003$ & $0.877 \pm 0.038$ \\
\hline & Accu $(\%)$ & $84.12 \pm 2.03$ & $81.95 \pm 1.87$ & $84.02 \pm 4.71$ & $83.00 \pm 1.03$ & $81.97 \pm 2.32$ \\
\hline & Sens $(\%)$ & $85.29 \pm 8.66$ & $86.94 \pm 5.97$ & $86.29 \pm 7.15$ & $81.13 \pm 1.33$ & $83.87 \pm 9.50$ \\
\hline & Spec $(\%)$ & $83.13 \pm 8.00$ & $77.32 \pm 2.23$ & $81.67 \pm 10.36$ & $84.73 \pm 1.58$ & $80.00 \pm 9.43$ \\
\hline
\end{tabular}

$\overline{\bar{a}}$ Definitions of performance figures. TP, TN, FP, and FN: the numbers of true positive, true negative, false positive, and false negative predicted by each algorithm, respectively; ROC $A_{z}$ : the area under the ROC curve; Accu: abbreviation of accuracy $=(\mathrm{TP}+\mathrm{TN}) /(\mathrm{TP}+\mathrm{TN}+\mathrm{FP}+\mathrm{FN})$; Sens: abbreviation of sensitivity $=\mathrm{TP} /(\mathrm{TP}$ $+\mathrm{FN})$; and Spec: abbreviation of specificity $=\mathrm{TN} /(\mathrm{TN}+\mathrm{FP})$.

texture features. On the other hand, it can be shown that using the hepatic features alone was inferior to the proposed approach (one tailed $p$ value $=0.005<0.05$ ). Moreover, Fig. 3 depicts the scatter plot of the mean contrasts of the highcontrast textons in the liver and spleen sonograms, i.e., (ACL, ACS), for one set of ROIs used in assessment III. One can see that a better separation may be achieved by using ACL and ACS together than by using ACL alone. This suggests that using the spleen as a reference could indeed improve the classification performance.

\section{DISCUSSION}

Diagnosis of liver cirrhosis based on sonograms highly depends on the experience of the sonologists. Different diagnoses may be made by different sonologists or by the same sonologist but at different times. To demonstrate the potential advantage of using the computer to assist in diagnosis of liver cirrhosis, two attending physicians with one and five experiences in liver sonography were asked to classify all images used in this study into three categories: cirrhotic, noncirrhotic, and equivocal. As shown in Table III, both observers achieved classification accuracies of approximately $80 \%$, with interobserver variability of $24.36 \%$. Moreover, both observes tended to achieve a better specificity than sensitivity. These results clearly suggest that there is still room for further improvement in classification of liver cirrhosis based on liver sonograms. The use of CAD approaches may bring about this improvement.

The unique characteristic of the proposed spleenreferenced approach lies in its normalization notion built into

TABLE VI. The coefficients of variation of $A_{z}$ values estimated in the four assessments for all five algorithms on the three ultrasound imaging systems.

\begin{tabular}{|c|c|c|c|c|c|}
\hline \multirow[b]{2}{*}{ Algorithms } & \multicolumn{3}{|c|}{ Assessments I and II } & \multirow{2}{*}{$\frac{\text { Assessment III }}{\text { All Systems }}$} & \multirow{2}{*}{$\frac{\text { Assessment IV }}{\text { Aloka-4000 }}$} \\
\hline & Toshiba-370A & Toshiba-380A & Aloka-4000 & & \\
\hline Proposed $(\%)$ & 0.31 & 1.27 & 2.72 & 0.77 & 0.82 \\
\hline Ogawa & 3.56 & 1.55 & 2.97 & 2.97 & 2.75 \\
\hline Modified Ogawa (\%) & 2.23 & 0.71 & 2.76 & 1.21 & 2.55 \\
\hline Yeh $(\%)$ & 4.54 & 1.56 & 3.82 & 1.66 & 4.24 \\
\hline Modified Yeh (\%) & 1.78 & 2.11 & 3.76 & 0.39 & 4.30 \\
\hline
\end{tabular}




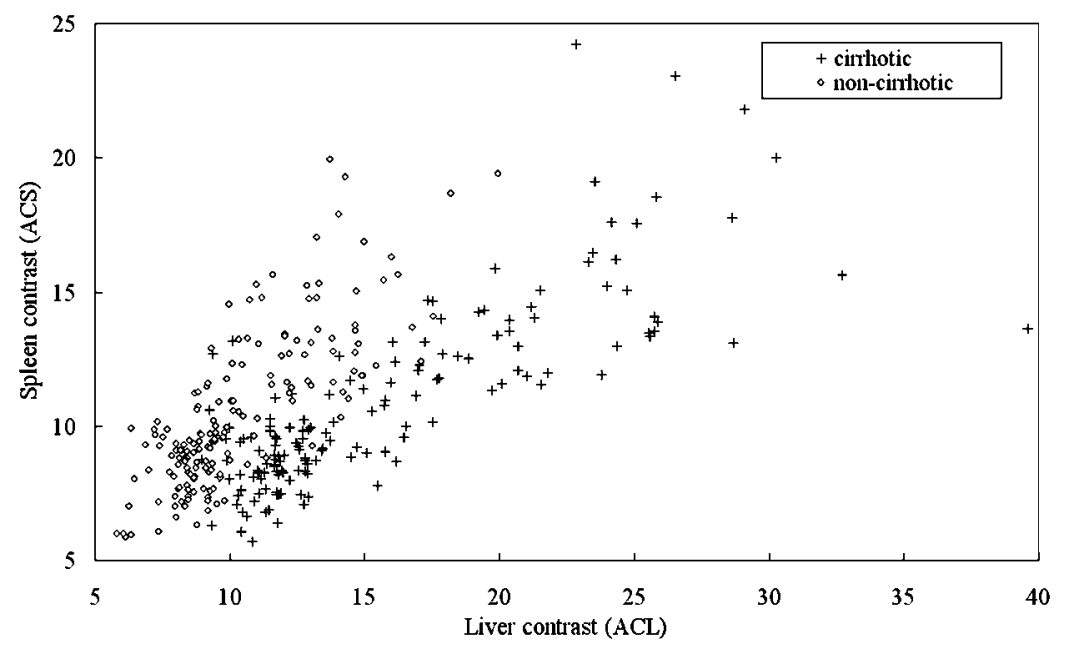

FIG. 3. The scatter plot of the mean contrasts of the high-contrast textons in the liver and spleen sonograms, i.e., (ACL, ACS), for one set of ROIs used in the third assessment. One can see that a better separation may be achieved by using ACL and ACS together than by using ACL alone. This suggests that using the spleen as a reference could indeed improve the classification performance. the four mathematical feature pairs, including one regionbased feature and three texton-based features. It not only alleviates the potential setting- and person-dependence problems, but also provides a better separation for the cirrhotic and noncirrhotic classes than using the hepatic features only. For example, in Fig. 3, the cirrhotic and noncirrhotic classes, which substantially overlap along the ACL dimension, tend to be spread out in the opposite directions along the ACS dimension, which aids in differentiation of both classes.

The performance of the proposed spleen-referenced approach was validated by four assessments to account for the effects of three factors, namely ROIs, ultrasound imaging systems, and scanned images. Table IV shows that the proposed algorithm was able to achieve reasonably high effectiveness and robustness in all cases tested when varying these three factors. Regardless of the variations in the ROIs, the means of the $A_{z}$ values and the best classification accuracies derived in assessments I and II are higher than 0.949 and $91.58 \%$, respectively, for all three systems. The CVs are smaller than $2.72 \%$ for all systems. For assessment III, the means of the $A_{z}$ values and the best classification accuracies are as high as 0.965 and $92.70 \%$, respectively, and the $\mathrm{CV}$ is as low as $0.77 \%$. For assessment IV, the means of the $A_{z}$ values and the classification accuracies are as high as 0.975 and $95.08 \%$, respectively, and the CV is only $0.83 \%$ on the Aloka SSD-4000.

Table V shows that the original and modified versions of the Ogawa algorithm have similar effectiveness with the mean $A_{z}$ values and best classification accuracies ranging from 0.844 to 0.919 and from $79.50 \%$ to $87.16 \%$, respectively. However, the original Ogawa algorithm tends to have a higher CV than the modified version. While these two algorithms have reasonably good performance for each system, they have relatively low accuracies in assessment III. For the original and modified versions of the Yeh algorithm, the mean $A_{z}$ values and best classification accuracies range from 0.871 to 0.896 and from $80.75 \%$ to $86.07 \%$, respectively. Both algorithms have similar CVs in general, except that the original one has a much higher $\mathrm{CV}$ than the modified version for the Toshiba SSA-370A.
In comparison with the original and modified versions of the Ogawa algorithm and the Yeh algorithm, the $p$ values for most one-tailed paired-sample $t$ tests are less than the individual significance level. It thus concludes that the proposed CAD algorithm is better than its counterparts at a $5 \%$ familywise significance level in terms of the algorithm effectiveness. The only two exceptions might be partially due to the relatively large standard deviations of these two algorithms on the images from the Aloka SSD-4000 (see Table V).

The proposed CAD algorithm also tends to be more robust than the other four algorithms, in terms of the $\mathrm{CV}$ values summarized in Table VI. Out of the five sets of $A_{z}$ values achieved in the four assessments, the proposed CAD algorithm has the smallest CVs for three sets and the second smallest for two sets, referring to the columns headed by "Toshiba-380A" and "All systems" in Table VI. In particular, with four scanned images acquired from each subject and one ROI taken from each image, the CV attained by the proposed algorithm is much smaller than that attained by the other four algorithms. This suggests that the proposed algorithm is more robust or equally robust to the variations of ROIs, ultrasound imaging systems, and scanned images in comparison with the other four algorithms.

One possible way to further evaluate the effect of using the spleen as a reference was to incorporate splenic features into the Ogawa algorithm and the Yeh algorithm. However, it was generally unfeasible in this study because both algorithms required a ROI of $64 \times 64$ pixels and we could not find such a large homogeneous region at the same depth as the liver sonograms in many spleen sonograms.

The idea of using spleen as a reference has been designed mainly to remedy the potential effects caused by different parameter settings, persons, and machines. It does not guarantee to work well for the mathematical features other than the proposed ones because the factors that degrade the performance of a feature may be more than setting, person, and machine dependence. Some mathematical features may simply fail to capture the sonographic features that can tell the difference between the cirrhotic and noncirrhotic livers. Incorporating splenic features would not augment differentiat- 
ing power in this case. Some mathematical features may depend on some other factors in addition to the parameter settings, persons, and machines. For example, for a liver sonogram acquired by a convex probe, e.g., PVM-375AT, the speckle patterns on the left and right sides of the image tend to have different orientations. If co-occurrence matrix based features are extracted, given $(d, \theta)$, the co-occurrence matrices for the ROIs on both sides of the image may be quite different due to the different speckle orientations. This effect may not be corrected by using the spleen as a reference.

One factor that might influence the performance of the proposed algorithm is the choice of the parameter values used in extracting the feature pairs. For example, for region growing of the VM map (block R2 in Fig. 1), if the threshold is too large or the maximum growing radius is too small, there would be little difference between the feature pairs, (ARSL, ARSS), of the cirrhotic and noncirrhotic subjects. It would greatly reduce the differentiating power of this feature pair. While the optimal parameter values may vary with the ROIs, the images, and the systems, we suggest estimating the parameter values by using a learning set of a small number of sonograms. The best way is to have a learning set for each system to account for the system-dependent nature of the parameter values and optimize the parameter values by taking into account all parameters involved in the four feature pairs simultaneously.

If the same set of parameter values is to be used by multiple systems because some systems do not have a sufficient number of subjects to form a learning set as in our case (Toshiba SSA-370A and Aloka SSD-4000), it is suggested that the parameter values be optimized for each feature pair separately. It is to avoid overfitting the parameter values to a specific system. By using this approach, we have obtained reasonably high performances for Toshiba SSA-370A and Aloka SSD-4000. Since these parameter values are not optimized globally, a slight perturbation in these values would not necessarily degrade the performance of the proposed algorithm significantly. For instance, if we change the threshold and the maximum growing radius specified in block R2 of Fig. 1 from $5 \%$ and 5 pixels to $6 \%$ and 4 pixels, the $A_{z}$ values of the Toshiba SSA-370A, Toshiba SSA-380A, and Aloka SSD-4000 become 0.994 $\pm 0.004,0.945 \pm 0.014$, and $0.978 \pm 0.025$, respectively. These changes are clearly insignificant (two tailed $p$ values $<0.05$ ). It is expected that the performance of the proposed algorithm may be further optimized for each system by using the learning set of its own.

Recently, it has been reported that Gamna-Gandy bodies may be observed in the spleen of a patient with liver cirrhosis. $^{24}$ Since Gamna-Gandy bodies usually appear as high-contrast calcified spots in the spleen of a cirrhotic subject, it should increase ACS if they are observable in the spleen sonogram. While it is likely that the performance of the proposed CAD algorithm would be degraded by the existence of Gamna-Gandy bodies, we had no clear evidence to ascribe any performance degradation to Gamna-Gandy bodies. It might be that because the spleen sonograms were acquired by the same system settings as the liver sonograms of the same subject, Gamna-Gandy bodies were not clearly visible in the images. Nevertheless, any splenic abnormality that would change the coarseness, contrast and texton sizes of splenic textures might degrade the performance of the proposed CAD algorithm, which would be a potential disadvantage of a spleen-referenced approach.

With the reasonably high effectiveness and robustness to the variations of ROIs, ultrasound imaging systems, and scanned images, one possible application of the proposed CAD algorithm is to assist the medical doctors to enhance the diagnosis accuracy of liver cirrhosis and reduce the interobserver variability. Since intraobserver variability was not investigated in this study, it is not clear if the proposed $\mathrm{CAD}$ algorithm is potentially valuable in reducing the intraobserver variability, which is a limitation of this study.

\section{CONCLUSIONS}

A new CAD algorithm was proposed in this study to assist in diagnosis of liver cirrhosis based on liver and spleen sonograms. It has been shown to be not only effective and robust to the variations of the ROIs, ultrasound imaging systems, and scanning views, but also superior to two previous CAD algorithms. Moreover, Table III shows that the classification accuracies attained by two attending physicians were around $80 \%$ when only liver and spleen sonograms were present. In comparison with human performance, as shown in Table IV, the classification accuracies achieved by the proposed CAD algorithm were higher than $90 \%$ for all assessments performed. It suggested that the proposed CAD algorithm would be beneficial, at least to less-experienced medical doctors, in differential diagnosis of cirrhotic and noncirrhotic livers.

\section{ACKNOWLEDGMENT}

This work was supported by National Science Council of Taiwan under Grant No. NSC94-2213-E-002-117.

\footnotetext{
${ }^{a)}$ Electronic mail: chung@ntu.edu.tw

${ }^{1}$ E. Arias and B. L. Smith, "Deaths: Preliminary data for 2001," Natl. Vital Stat. Rep. 51, 1-5 (2003).

${ }^{2} \mathrm{DOH}$ Taiwan, http://www.doh.gov.tw/dohenglish/Upload/Statistics/S02/ 9101-eng.xls, Statistics for causes of death 2002, Department of Health, Taiwan.

${ }^{3}$ P. M. Yang et al., "Ultrasonography in the diagnosis of benign diffuse parenchymal liver diseases: A prospective study," J. Formos. Med. Assoc. 87, 966-977 (1988).

${ }^{4}$ A. E. A. Joseph, S. H. Saverymuttu, S. Al-Sam, M. G. Cook, and J. D. Maxwell, "Comparison of liver histology with ultrasonography in assessing diffuse parenchymal liver disease," Clin. Radiol. 43, 26-31 (1991).

${ }^{5}$ S. Gaiani et al., "What is the criterion for differentiating chronic hepatitis from compensated cirrhosis? A prospective study comparing ultrasonography and percutaneous liver biopsy," J. Hepatol. 27, 979-985 (1997).

${ }^{6}$ H. Tchelepi, P. W. Ralls, R. Radin, and E. Grant, "Sonography of diffuse liver disease," J. Ultrasound Med. 21, 1023-1032 (2002).

${ }^{7}$ V. Vilgrain, D. Lebrec, Y. Menu, A. Scherrer, and H. Nahum, "Comparison between ultrasonographic signs and the degree of portal hypertension in patients with cirrhosis," Gastrointest. Radiol. 15, 218-222 (1990).

${ }^{8} \mathrm{~J}$. S. Bleck et al., "Random field models in the textural analysis of ultrasonic images of the liver," IEEE Trans. Med. Imaging 15, 796-801 (1996).

${ }^{9}$ Y. M. Kadah, A. A. Farag, J. M. Zurada, A. M. Badawi, and A. M. Youssef, "Classification algorithms for quantitative tissue characterization of diffuse liver disease from ultrasound images," IEEE Trans. Med. Imaging 15, 466-478 (1996).
} 
${ }^{10}$ K. Ogawa, M. Fukushima, K. Kubota, and N. Hisa, "Computer-aided diagnostic system for diffuse liver diseases with ultrasonography by neural networks," IEEE Trans. Nucl. Sci. 45, 3069-3074 (1998).

${ }^{11}$ M. H. Horng, Y. N. Sun, and X. Z. Lin, "Texture feature coding method for classification of liver sonography," Comput. Med. Imaging Graph. 26, 33-42 (2002).

${ }^{12}$ A. Mojsilovic, M. Popovic, S. Markovic, and M. Krstic, "Characterization of visually similar diffuse diseases from B-scan liver images using nonseparable wavelet transform," IEEE Trans. Med. Imaging 17, 541549 (1998).

${ }^{13}$ B. J. Oosterveld, J. M. Thijssen, R. L. Hartman, R. L. Romijn, and G. J. E. Rosenbusch, "Ultrasound attenuation and texture analysis of diffuse liver disease: Methods and preliminary results," Phys. Med. Biol. 36, 1039-1064 (1991).

${ }^{14}$ M. Lang, H. Ermert, and L. Heuser, "In vivo study of on line liver tissue classification based on envelope power spectrum analysis," Ultrason. Imaging 16, 77-86 (1994).

${ }^{15}$ T. Kikuchi et al., "Development of a new diagnostic system for human liver diseases based on conventional ultrasonic diagnostic equipment," Jpn. J. Appl. Phys., Part 1 40, 3888-3889 (2001).

${ }^{16}$ W. R. Dillon and M. Goldstein, Multivariate Analysis: Method and Applications (Wiley, New York, 1984).
${ }^{17} \mathrm{~K}$. Ishak et al., "Histological grading and staging of chronic hepatitis," J. Hepatol. 22, 696-699 (1995).

${ }^{18}$ T. Loupas, W. N. McDicken, and P. L. Allan, "An adaptive weighted median filter for speckle suppression in medical ultrasonic image," IEEE Trans. Circ. Syst. 36, 129-135 (1989).

${ }^{19}$ M. Karaman, M. A. Kutay, and G. Bozdagi, "An adaptive speckle suppression filter for medical ultrasound imaging," IEEE Trans. Med. Imaging 14, 283-292 (1995).

${ }^{20}$ X. Hao, S. Gao, and X. Gao, "A novel multiscale nonlinear thresholding method for ultrasonic speckle suppressing," IEEE Trans. Med. Imaging 18, 787-794 (1999).

${ }^{21}$ Y. Chen, R. Yin, P. Flynn, and S. L. Broschat, "Aggressive region growing for speckle reduction in ultrasound images," Pattern Recognit. Lett. 24, 677-691 (2003).

${ }^{22}$ L. Vincent and P. Soille, "Watershed in digital spaces: An efficient algorithm based on immersion simulations," IEEE Trans. Pattern Anal. Mach. Intell. 13, 583-597 (1991).

${ }^{23}$ W. C. Yeh, S. H. Huang, and P. C. Li, "Liver fibrosis grade classification with B-mode ultrasound," Ultrasound Med. Biol. 29, 1229-1235 (2003).

${ }^{24}$ M. J. Chen et al., "Ultrasonography of splenic abnormalities," World J. Gastroenterol. 11, 4061-4066 (2005). 